\title{
Pioglitazone prevents sevoflurane-induced neuroinflammation and cognitive decline in a rat model of chronic intermittent hypoxia by upregulating hippocampal PPAR- $\gamma$
}

\author{
XIYAN ZHANG ${ }^{1}$, NING LI ${ }^{2}$, LINGLING LU ${ }^{3}$, QUAN LIN ${ }^{4}$, LIANG LI $^{1}$, PING DONG ${ }^{1}$, \\ BO YANG ${ }^{1}$, DONGLIANG LI ${ }^{1}$ and JIANCHUN FEI ${ }^{1}$ \\ ${ }^{1}$ Department of Anesthesiology, Qilu Hospital, Shandong University, Jinan, Shandong 250012; \\ ${ }^{2}$ School of Public Health, Jining Medical University; ${ }^{3}$ Shandong Province Jining Health School, Jining, Shandong 272067; \\ ${ }^{4}$ Shengli Oilfield Central Hospital, Dongying, Shandong 257000, P.R. China
}

Received July 25, 2018; Accepted March 8, 2019

DOI: $10.3892 / \mathrm{mmr} .2019 .10052$

\begin{abstract}
Post-operative cognitive dysfunction is a common complication after anesthesia and surgery. Sevoflurane (SEV), a widely used inhalational anesthetic, can exaggerate neuroinflammation and cause cognitive dysfunction under chronic intermittent hypoxia $(\mathrm{CIH})$ conditions by downregulating hippocampal peroxisome proliferator-activated receptor- $\gamma($ PPAR- $\gamma)$. In the present study, it was examined whether treatment with PPAR- $\gamma$ agonist pioglitazone (PIO) is beneficial in counteracting SEV-induced neuroinflammation and cognitive decline in a rat model of CIH. Rats were exposed to $\mathrm{CIH}$ for 4 weeks. After 2 weeks of $\mathrm{CIH}$, these animals underwent either $2.6 \% \mathrm{SEV}$ or control (CON) exposure for $4 \mathrm{~h}$. PIO $(60 \mathrm{mg} / \mathrm{kg})$ or vehicle (VEH) was administered orally twice daily for 2 weeks, starting one day prior to SEV or CON exposure. Compared with $\mathrm{CIH}-\mathrm{CON}+\mathrm{VEH}$ rats, $\mathrm{CIH}-\mathrm{SEV}+\mathrm{VEH}$ rats exhibited significant cognitive decline as indicated by increased latency to locate the hidden platform and shorter dwell-time in the goal quadrant in the Morris Water Maze task. Molecular studies revealed that CIH-SEV+VEH rats had increased proinflammatory cytokine expression and microglial activation in the hippocampus, which were associated with decreased PPAR- $\gamma$ activity. Notably, SEV-induced cognitive decline and increases in proinflammatory cytokine expression and microglial activation were prevented by PIO, which increased hippocampal PPAR- $\gamma$ activity. PIO also increased hippocampal PPAR- $\gamma$ activity in CIH-CON rats but did not alter proinflammatory cytokine expression and microglial activation as well as cognitive function. Additionally,
\end{abstract}

Correspondence to: Dr Jianchun Fei, Department of Anesthesiology, Qilu Hospital, Shandong University, 107 Wenhua Xi Road, Jinan, Shandong 250012, P.R. China

E-mail: fei-jc@hotmail.com

Key words: sevoflurane, pioglitazone, chronic intermittent hypoxia, cognitive dysfunction, neuroinflammation expression of hippocampal PPAR- $\alpha$ and PPAR- $\beta$, two other PPAR isotypes, were comparable among the groups. These data suggest that PIO prevents SEV-induced exaggeration of neuroinflammation and cognitive decline under $\mathrm{CIH}$ conditions by upregulating hippocampal PPAR- $\gamma$. PIO may have the potential to prevent anesthetic SEV-induced cognitive decline in surgical patients with obstructive sleep apnea.

\section{Introduction}

Post-operative cognitive dysfunction (POCD) generally refers to the decline in cognitive ability after anesthesia and surgery when compared to preoperative cognitive status (1). POCD can occur in nearly $10 \%$ of all surgical patients and $40 \%$ of elderly patients at the point of discharge, and the long-term impact of this is marked by a significantly higher mortality rate than age and sex-matched controls without POCD (1). Although the causes of POCD are not fully understood, accumulating evidence from experimental and clinical studies has shown that general anesthesia alone is capable of causing cognitive dysfunction (2). It is worth noting that anesthesia-induced cognitive dysfunction may depend on the types of anesthetic agents, drug doses, exposure duration and patient age $(3,4)$. In addition, the presence of a pathological condition may increase the susceptibility to developing POCD or exacerbate the pre-existing cognitive impairment after anesthesia (5-7). Neuroinflammation in the brain, particularly in the hippocampus, has been shown to play a contributory role in the pathogenesis of cognitive dysfunction, including POCD (8-10). Microglia, the resident innate immune cells in the brain, are major sources of pro-inflammatory cytokines (11). Activation of microglia in the brain results in the release of pro-inflammatory cytokines, which triggers the neuroinflammatory response and subsequently leads to neuronal damage and losses (11-13). Interventions that inhibit microglia activity and neuroinflammation in the brain can significantly ameliorate cognitive dysfunction in multiple neurodegenerative diseases $(8,14)$.

Obstructive sleep apnea, characterized by repeated occlusions of the pharyngeal airway during sleep, is a devastating 
respiratory control disorder and is associated with serious and adverse consequences including cognitive dysfunction and dementia $(15,16)$. It is widely accepted that chronic intermittent hypoxia $(\mathrm{CIH})$, a cardinal feature of obstructive sleep apnea, plays an important role in cognitive dysfunction in obstructive sleep apnea (17). CIH can induce activation of microglia and subsequent neuroinflammation in the hippocampus, a key region of the brain associated with spatial learning and memory acquisition, contributing to cognitive dysfunction $(15,18)$.

Peroxisome proliferator-activated receptor- $\gamma$ (PPAR- $\gamma$ ) belongs to the PPAR family of ligand-activated transcription factors that is well-known to regulate adipocyte differentiation, fatty acid storage and glucose metabolism (19). In addition, PPAR- $\gamma$ plays an important role in the immune response through its ability to inhibit the expression of inflammatory cytokines and to direct the differentiation of immune cells towards anti-inflammatory phenotypes (20-22). In the brain, activation or upregulation of PPAR- $\gamma$ has been demonstrated to inhibit the synthesis and release of pro-inflammatory cytokines in many central nervous system diseases in which massive inflammation plays a detrimental role (23-25). Notably, PPAR- $\gamma$ is expressed in multiple cell types in the brain including microglia, astrocytes and neurons $(26,27)$, and relatively high PPAR- $\gamma$ expression levels have been found in the brain areas associated with learning and memory including the hippocampus $(26,27)$. Sevoflurane (SEV) is one of the most widely used anesthetic agents for induction and maintenance of general anesthesia in surgical patients, including patients with obstructive sleep apnea. Studies from our laboratory and others have recently demonstrated that a moderate duration of SEV (2-3\% for 2 or $4 \mathrm{~h}$ ) does not cause neuroinflammation in the hippocampus and cognitive impairment in either adult or aged animals (4,28-30). However, SEV downregulates PPAR- $\gamma$ expression and activity in the hippocampus, which increase the sensitivity and susceptibility to $\mathrm{CIH}$ insult, leading to aggravated microglia activation and neuroinflammation as well as exaggeration of cognitive decline under CIH conditions (28). Pioglitazone (PIO) is a thiazolidinedione (TZD) class synthetic PPAR $-\gamma$ agonist and has been used for the treatment of patients with type II diabetes mellitus. In addition, PIO exhibits anti-inflammatory and neuroprotective effects in multiple inflammatory central nervous system disorders $(31,32)$. PIO can cross the blood-brain barrier (33). The present study was designed to examine whether treatment with PPAR- $\gamma$ agonist PIO would upregulate PPAR- $\gamma$ expression and activity in the hippocampus, preventing SEV-induced cognitive decline in a rat model of $\mathrm{CIH}$.

\section{Materials and methods}

Animals. A total of 60 male Sprague-Dawley rats weighing 200-250 g (8-9 weeks of age) were purchased from Beijing Laboratory Animal Research Center (Beijing, China) and maintained on a 12:12 h light/dark cycle at room temperature $\left(23 \pm 2^{\circ} \mathrm{C}\right)$ in $50-60 \%$ relative humidity with ad libitum access to food and water. All experimental protocols were approved by the Animal Care and Use Committees of Shandong University and conducted in accordance with the guidelines of the Animal Care and Use Committee at Shandong University (Jinan, Shandong, China).
Protocol. Rats were placed into a plastic cage equipped with the intermittent hypoxia apparatus and exposed to intermittent hypoxia for 4 weeks, as previously described (28). The hypoxia/re-oxygenation profile was run for eight consecutive hours during the $12 \mathrm{~h}$ of light cycle each day to coincide with the animal sleep cycle. During each cycle of intermittent hypoxia, the oxygen concentration in the cage dropped from 21 to $5 \%$ over a 50 -sec period and was then quickly returned to $21 \%$ during the following $40 \mathrm{sec}$. Constant room air was given to the cages during the $12 \mathrm{~h}$ of the dark cycle each day. After 2 weeks of intermittent hypoxia, CIH rats underwent either control (CON, room air) or a moderate duration of SEV (2.6\%) exposure for $4 \mathrm{~h}$, and were treated with vehicle (VEH, distilled water) or PPAR- $\gamma$ agonist PIO for 2 weeks, starting one day prior to $\mathrm{CON}$ or SEV exposure. The experimental groups were as follows ( $\mathrm{n}=15$ for each group): i) $\mathrm{CIH}-\mathrm{CON}+\mathrm{VEH}$, ii) $\mathrm{CIH}-\mathrm{CON}+\mathrm{PIO}$, iii) $\mathrm{CIH}-\mathrm{SEV}+\mathrm{VEH}$, and iv) $\mathrm{CIH}-\mathrm{SEV}+\mathrm{PIO}$. SEV exposure was conducted as previously described (28). Briefly, animals assigned to SEV exposure were placed in a temperature-controlled chamber that was equipped with an anesthesia device and a multi-gas monitor. SEV $(2.6 \%)$ was provided by a humidified $30 \% \mathrm{O}_{2}$ carrier gas from a calibrated vaporizer for $4 \mathrm{~h}$. Animals assigned to $\mathrm{CON}$ exposure were also placed in the same chamber for $4 \mathrm{~h}$ but no SEV was provided. After CON or SEV exposure, all animals were returned to their original cages and underwent $\mathrm{CIH}$ for another 2 weeks. PIO $(60 \mathrm{mg} / \mathrm{kg})$ or VEH was administered twice daily by oral gavage, as described previously (31). At the end of the study protocol, spatial learning and memory in some animals ( $\mathrm{n}=9$ for each group) were examined using the Morris Water Maze test. Immediately after Morris Water Maze examination, some of the animals were sacrificed by decapitation under deep pentobarbital anesthesia and brains were quickly collected for molecular analyses. The rest of the animals ( $n=6$ for each group) were transcardially perfused with saline containing heparin $(1 \mathrm{U} / \mathrm{ml})$ and then with $4 \%$ paraformaldehyde in $0.1 \mathrm{M}$ phosphate buffer (PB) for immunofluorescent study. The experimental procedures and the timeline are shown in Fig. 1A.

Morris water maze task. Spatial learning and memory were examined using the Morris Water Maze after 4 weeks of intermittent hypoxia, as previously described (28). The spatial acquisition trial was performed over four consecutive days and each acquisition trial section consisted of four trials with an interval of $15 \mathrm{~min}$. A rat was placed gently into the water facing the wall of the pool and allowed $120 \mathrm{sec}$ to find a hidden escape platform submerged approximately $1 \mathrm{~cm}$ below the water surface. The time for each rat spent to locate the submerged platform was recorded as the escape latency. A probe trial was conducted $24 \mathrm{~h}$ after completion of the spatial acquisition trial to assess the spatial reference memory. During the probe test, the platform was removed from the pool and rats were allowed to swim freely for $120 \mathrm{sec}$ in any of the four quadrants of the swimming pool. The percentage of time spent in the target quadrant where the platform had been placed was calculated as a measure of memory for platform position.

Western blot analysis. The rat brains were dissected and bilateral hippocampal tissues were quickly removed. Hippocampal 
A

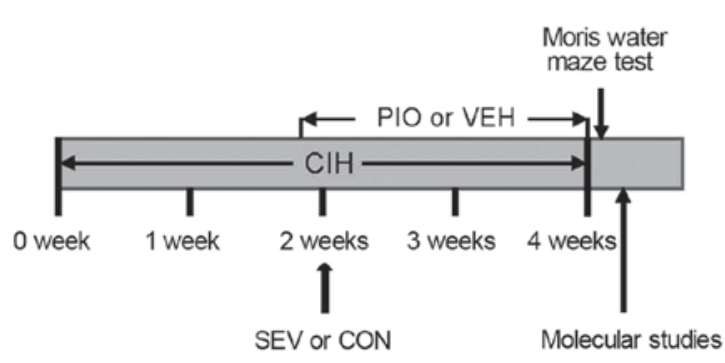

C

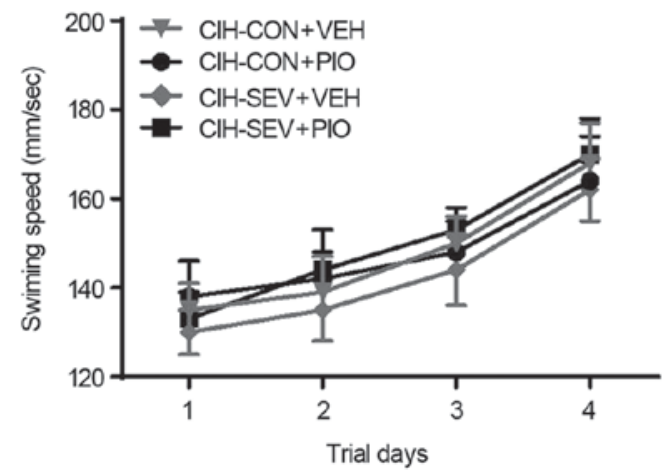

B
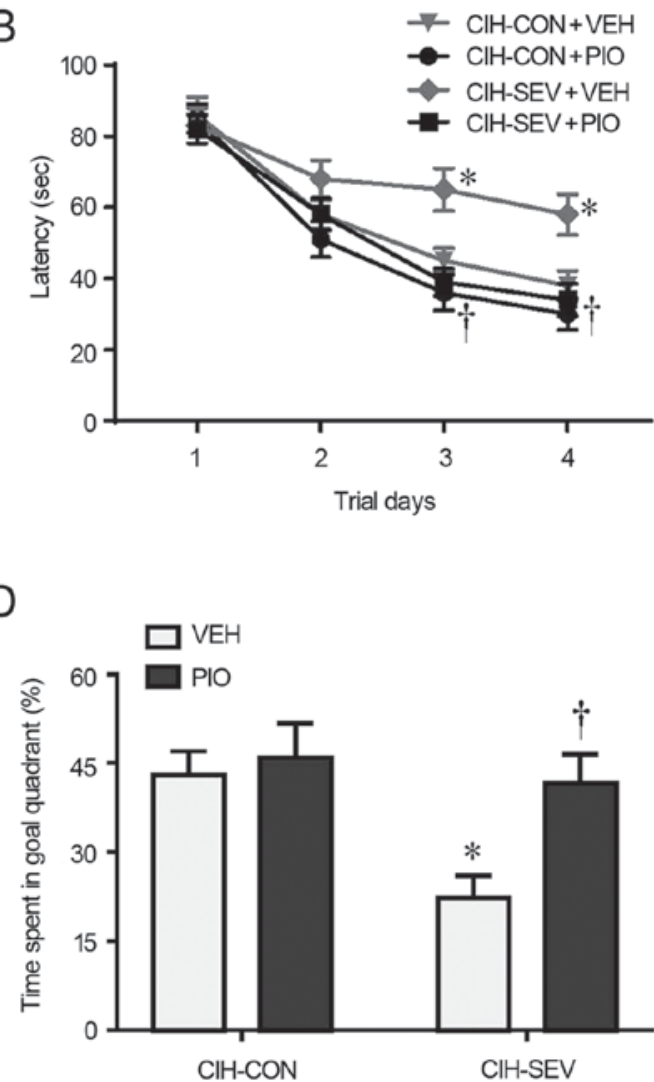

Figure 1. (A) Summary of the experimental procedures and timeline. (B-D) Effects of pioglitazone (PIO) treatment on sevoflurane (SEV)-induced cognitive decline in the CIH rats. Compared with the CIH-CON rats treated with VEH (CIH-CON+VEH), CIH-SEV rats treated with VEH (CIH-SEV+VEH) exhibited increased latency to locate the hidden platform (B) and shorter dwell-time in the goal quadrant (D) in Morris Water Maze task. PIO treatment reduced the escape latency and increased dwell-time in the goal quadrant in the CIH-SEV rats. Notably, the swimming speed was similar across the four experimental groups (C). Data are expressed as the mean \pm SEM ( $\mathrm{n}=9$ per group). ${ }^{*} \mathrm{P}<0.05$ vs. CIH-CON+VEH; ${ }^{\mathrm{P}}<0.05, \mathrm{CIH}-\mathrm{SEV}+\mathrm{PIO}$ vs. CIH-SEV+VEH. CIH, chronic intermittent hypoxia; CON, control; VEH, vehicle.

tissues were homogenized in cold lysis buffer (Beyotime Institute of Biotechnology, Shanghai, China) containing protease inhibitors. The proteins extracted from the hippocampal tissues were loaded onto $12 \%$ sodium dodecyl sulfate-polyacrylamide gel electrophoresis (SDS-PAGE) gel and then transferred to polyvinylidene fluoride membranes. The membranes were immunoblotted with primary antibodies to tumor necrosis factor (TNF)- $\alpha$ (1:200; sc-52746), interleukin (IL)-1 $\beta$ (1:200; sc-52012), PPAR- $\gamma$ (1:100; sc-7273), PPAR- $\alpha$ (1:100; sc-398394), PPAR- $\beta$ (1:200; sc-74517) and $\beta$-actin (1:1,000; sc-47778) (Santa Cruz Biotechnology, Inc., Santa Cruz, CA, USA) at $4^{\circ} \mathrm{C}$ overnight and then incubated with HRP-conjugated secondary antibodies (1:5,000; sc-2031) (Santa Cruz Biotechnology, Inc.) for $1 \mathrm{~h}$ at room temperature. The immunoreactive bands were visualized using enhanced chemiluminescence detection system (GE Healthcare, Chicago, IL, USA) and analyzed with ImageJ software version 1.49v (NIH; National Institutes of Health, Bethesda, MD, USA). All data were normalized to $\beta$-actin.

Determination of PPAR- $\gamma$ activity. Nuclear extracts were prepared from the hippocampal tissues with a Nuclear Extract Kit (Active Motif, Carlsbad, CA, USA). PPAR $-\gamma$ DNA binding activity was measured using Transcription Factor Assay Kit (Active Motif) following the manufacturer's instructions.
Immunofluorescence study. Immunofluorescence staining for microglia was performed as previously described (28). Briefly, perfused brains were removed and post-fixed in $4 \%$ paraformaldehyde at $4^{\circ} \mathrm{C}$ overnight followed by incubation in $30 \%$ sucrose for $48 \mathrm{~h}$ at $4^{\circ} \mathrm{C}$. After being embedded in optimal cutting temperature (OCT), the brains were serially sectioned at $20-\mu \mathrm{m}$ intervals using a cryostat microtome. The sections were immunostained at $4^{\circ} \mathrm{C}$ overnight with anti-CD11b primary antibody (MCA275R; Chemicon, Temecula, CA, USA) that recognizes both non-activated and activated microglia, and were then incubated with a secondary antibody (Alex Fluor 488; A-11001, Invitrogen Thermo Fisher Scientific, Inc., Waltham, MA, USA) for $2 \mathrm{~h}$ at room temperature. Fluorescence images were acquired using a Zeiss LSM 510 confocal microscope at 40x magnification and the number of total and activated microglia were counted in several $0.2 \times 0.2 \mathrm{~mm}$ squares. Activated microglia were presented as a percentage of the total number of microglia.

Statistical analysis. All data are presented as the mean \pm standard error of the mean. A two-way analysis of variance followed by Bonferroni's post hoc test was performed for statistical analysis using GraphPad Prism 7 (GraphPad Software, La Jolla, CA, USA). Statistical significance was accepted at $\mathrm{P}<0.05$. 
A

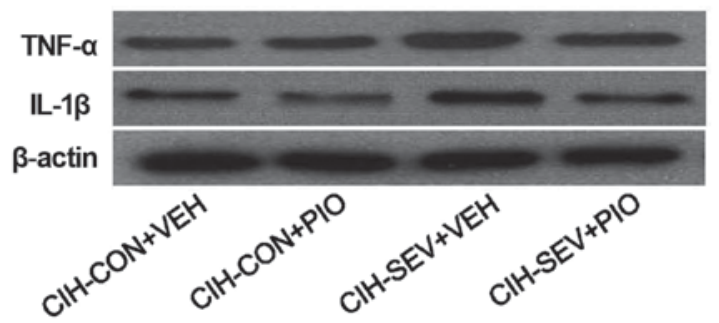

B

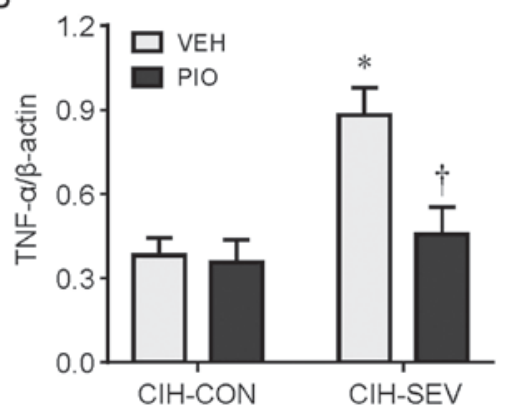

C

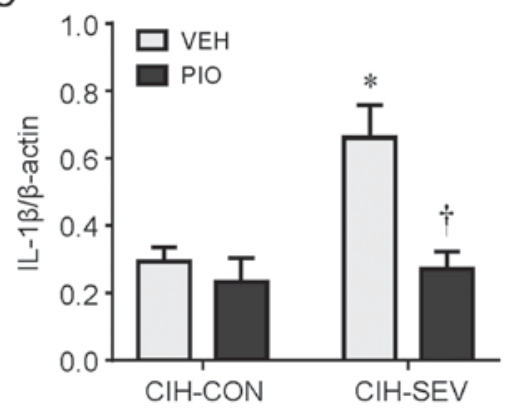

Figure 2. Effects of pioglitazone (PIO) treatment on sevoflurane (SEV)-induced neuroinflammation in the hippocampus in the CIH rats. Compared with the $\mathrm{CIH}-\mathrm{CON}$ rats treated with VEH (CIH-CON+VEH), CIH-SEV rats treated with VEH (CIH-SEV+VEH) had increased expression of TNF- $\alpha$ (A and B) and IL-1 $\beta$ (A and C), which was inhibited by PIO treatment. Data are expressed as the mean $\pm \mathrm{SEM}\left(\mathrm{n}=9 \mathrm{per}\right.$ group). ${ }^{*} \mathrm{P}<0.05$ vs. $\mathrm{CIH}-\mathrm{CON}+\mathrm{VEH}$; ${ }^{\dagger} \mathrm{P}<0.05$, $\mathrm{CIH}-\mathrm{SEV}+\mathrm{PIO}$ vs. CIH-SEV+VEH. CIH, chronic intermittent hypoxia; CON, control; VEH, vehicle.

\section{Results}

Effects of PIO treatment on SEV-induced cognitive decline. Four weeks after intermittent hypoxia and 2 weeks after CON or SEV exposure, the Morris Water Maze task was performed to assess spatial learning and reference memory in each mouse group. As shown in Fig. 1B, CIH-SEV rats treated with VEH (CIH-SEV+VEH) exhibited significantly longer latency to find the hidden platform on the last two trials when compared with the CIH-CON rats treated with $\mathrm{VEH}(\mathrm{CIH}-\mathrm{CON}+\mathrm{VEH})$. A 2-week PIO treatment, starting one day prior to CON or SEV exposure, did not alter the escape latency in the $\mathrm{CIH}-\mathrm{CON}$ rats, but it significantly reduced the escape latency in the CIH-SEV rats to an extent similar to that observed in the $\mathrm{CIH}-\mathrm{CON}$ rats. Notably, the swimming speed across four experimental groups was comparable throughout 4 consecutive days (Fig. 1C). Probe trial showed that the percent time spent in the target quadrant was markedly reduced in the CIH-SEV rats treated with VEH (CIH-SEV+VEH) as compared to the CIH-CON rats treated with VEH (CIH-CON+VEH) (Fig. 1D). Compared with the respective $\mathrm{VEH}$ groups, PIO treatment significantly increased the percent time spent in the target quadrant in the $\mathrm{CIH}-\mathrm{SEV}$ rats but not in the $\mathrm{CIH}-\mathrm{CON}$ rats.

Effects of PIO treatment on SEV-induced neuroinflammation in the hippocampus. To examine the effect of PIO treatment on neuroinflammation, the protein levels of TNF- $\alpha$ and IL-1 $\beta$, two key proinflammatory cytokines in the hippocampus, were assessed. Compared with the CIH-CON rats treated with VEH, CIH-SEV rats treated with $\mathrm{VEH}$ had significantly increased protein levels of both TNF- $\alpha$ (Fig. 2A and B) and IL-1 $\beta$ (Fig. 2A and C) in the hippocampus. Importantly, the increases in protein levels of TNF- $\alpha$ and IL-1 $\beta$ observed in the CIH-SEV rats were inhibited by PIO treatment, which did not alter these proinflammatory cytokines in the $\mathrm{CIH}-\mathrm{CON}$ rats.

Effects of PIO treatment on SEV-induced microglial activity in the hippocampus. Immunofluorescence study revealed that the number of total microglia in the hippocampus was comparable among the four experimental groups (Fig. 3A and B). However, the number of activated microglia that were distinguished by strong CD11b immunoreactivity, an enlarged cell body, and fewer and shorter hypertrophic processes extending from the soma, was markedly higher in the hippocampus in the CIH-SEV rats treated with VEH compared with CIH-CON rats treated with $\mathrm{VEH}$ (Fig. 3A and $\mathrm{C}$ ). Compared with the respective $\mathrm{VEH}$ groups, $\mathrm{PIO}$ treatment did not change the number of activated microglia in the $\mathrm{CIH}-\mathrm{CON}$ rats, but it significantly reduced the number of activated microglia in the CIH-SEV rats.

Effects of PIO treatment on PPAR isoform expression and activity in the hippocampus. To determine whether PIO treatment prevented exaggerated microglia activation and neuroinflammation by upregulating hippocampal PPAR- $\gamma$, hippocampal PPAR- $\gamma$ expression and activity were further evaluated. PPAR- $\gamma$ protein levels (Fig. 4A) and its DNA binding activity (Fig. 4B) in the hippocampus were significantly decreased in the CIH-SEV rats treated with $\mathrm{VEH}$ compared with the $\mathrm{CIH}-\mathrm{CON}$ rats treated with $\mathrm{VEH}$. Interestingly, PIO treatment markedly increased PPAR- $\gamma$ expression and its DNA binding activity in both the CIH-CON and CIH-SEV rats when compared with their respective $\mathrm{VEH}$ groups. Of note, there were no significant differences in expression of PPAR isoforms PPAR- $\alpha$ (Fig. 5A and B) or PPAR- $\beta$ (Fig. 5A and C) among the four experimental groups. 
A

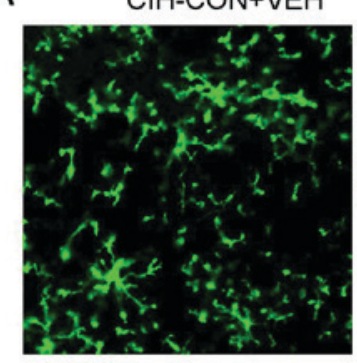

B

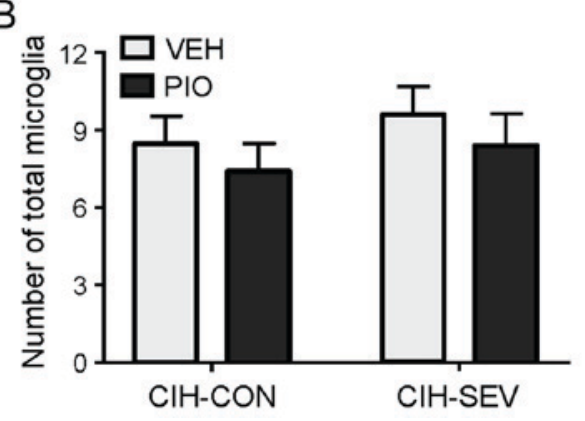

CIH-SEV+VEH
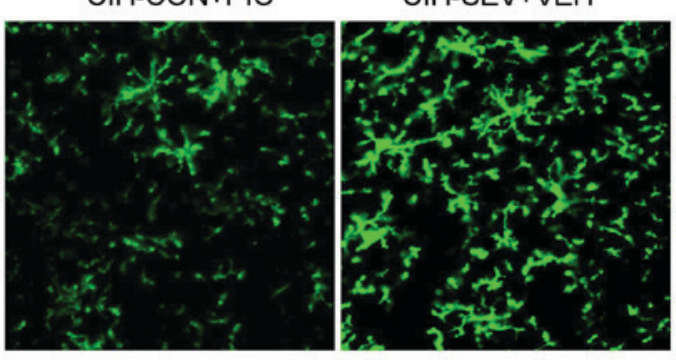

$\mathrm{C}$

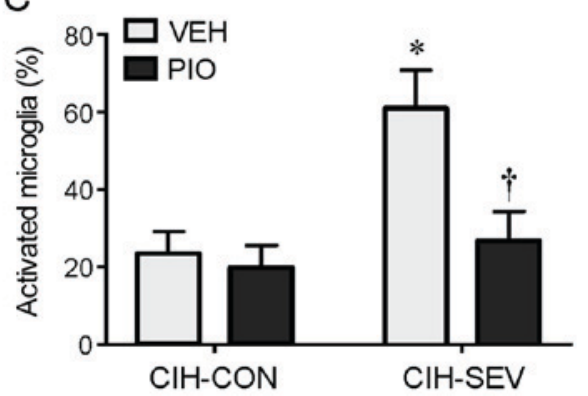

Figure 3. Effects of pioglitazone (PIO) treatment on sevoflurane (SEV)-induced microglial activity in the hippocampus in CIH rats. (A) Representative photomicrographs showing CD11b-immunoreactive microglia in the hippocampus in each group. Images were acquired at $\mathrm{x} 40$ magnification. (B) There was no difference in the total number of microglia across groups. (C) The number of activated microglia was higher in the $\mathrm{CIH}-\mathrm{SEV}$ rats treated with $\mathrm{VEH}$ but was reduced in the $\mathrm{CIH}-\mathrm{SEV}$ rats treated with $\mathrm{PIO}$. Data are expressed as the mean $\pm \mathrm{SEM}$ (n=6 per group). ${ }^{*} \mathrm{P}<0.05$ vs. $\mathrm{CIH}-\mathrm{CON}+\mathrm{VEH}$; $\mathrm{P}<0.05$, CIH-SEV+PIO vs. CIH-SEV+VEH. CIH, chronic intermittent hypoxia; CON, control; VEH, vehicle.
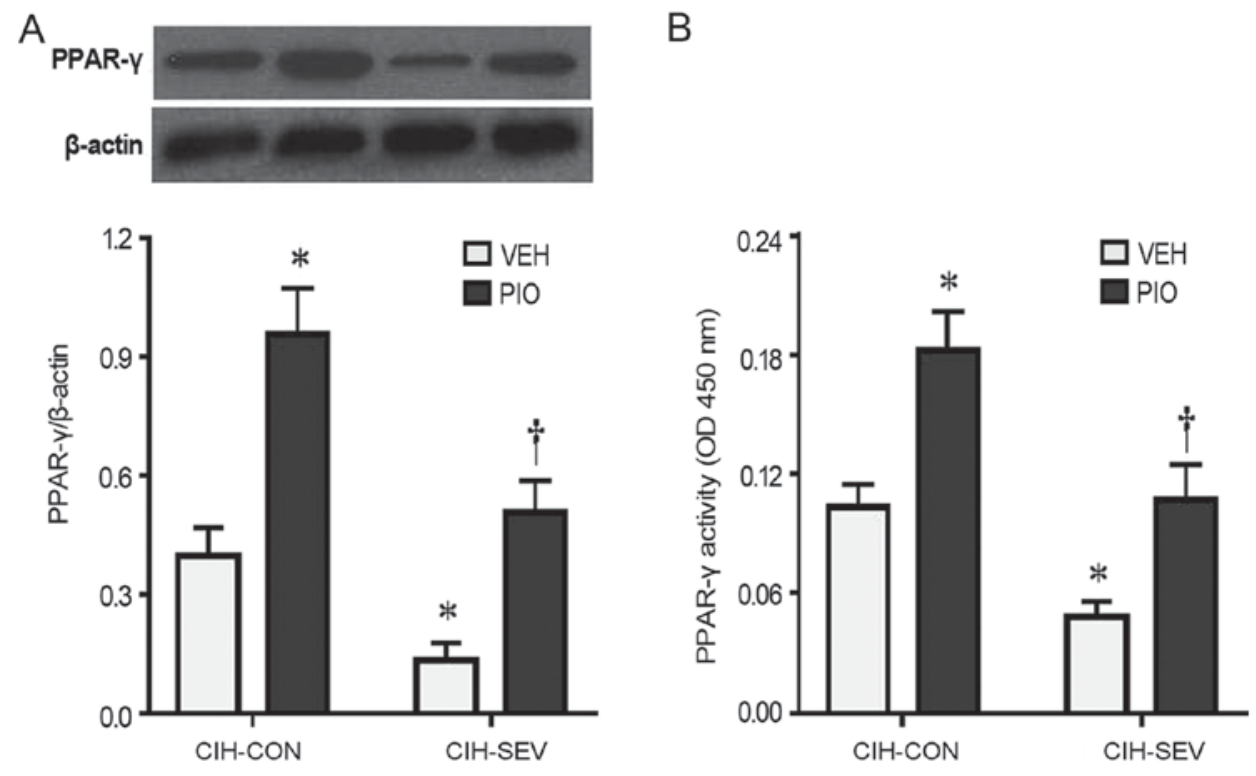

Figure 4. Effects of pioglitazone (PIO) treatment on expression (A) and activity (B) of PPAR- $\gamma$ in the hippocampus in the CIH rats. Compared with the CIH-CON rats treated with VEH, CIH-SEV rats treated with VEH had decreased expression and activity of PPAR- $\gamma$. PIO treatment increased expression and activity of PPAR- $\gamma$ in both the $\mathrm{CIH}-\mathrm{CON}$ and $\mathrm{CIH}-\mathrm{SEV}$ rats. Data are expressed as the mean $\pm \mathrm{SEM}$ ( $\mathrm{n}=9 \mathrm{per}$ group). ${ }^{*} \mathrm{P}<0.05$ vs. $\mathrm{CIH}-\mathrm{CON}+\mathrm{VEH}$; ${ }^{\dagger} \mathrm{P}<0.05$, CIH-SEV+PIO vs. CIH-SEV+VEH. PPAR, peroxisome proliferator-activated receptor; CIH, chronic intermittent hypoxia; CON, control; VEH, vehicle.

\section{Discussion}

The major finding of the present study is that systemic administration of PPAR- $\gamma$ agonist PIO upregulated hippocampal PPAR $-\gamma$ expression and activity, inhibited anesthetic SEV-induced hippocampal microglia activation and neuroinflammation, and prevented SEV-induced cognitive decline in a rat model of $\mathrm{CIH}$.
Obstructive sleep apnea is increasing in prevalence although it still remains significantly underdiagnosed (34). Patients with obstructive sleep apnea subjected to surgery are at increased risk for a wide variety of postoperative complications including cognitive decline, which should be recognized and managed in the perioperative period to minimize postoperative complications. We and others have previously reported that exposure to a moderate duration of SEV in animals did not 


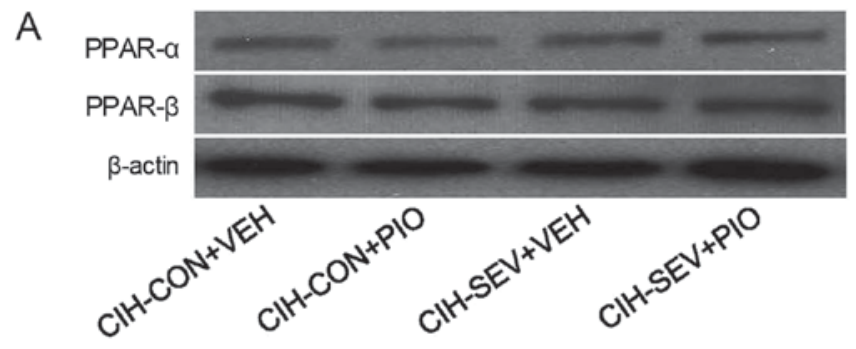

B

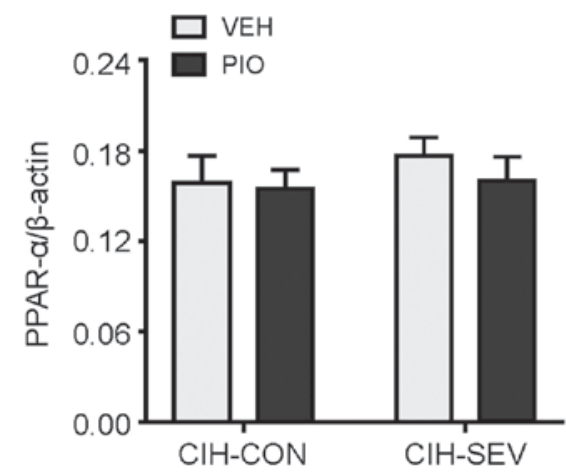

C

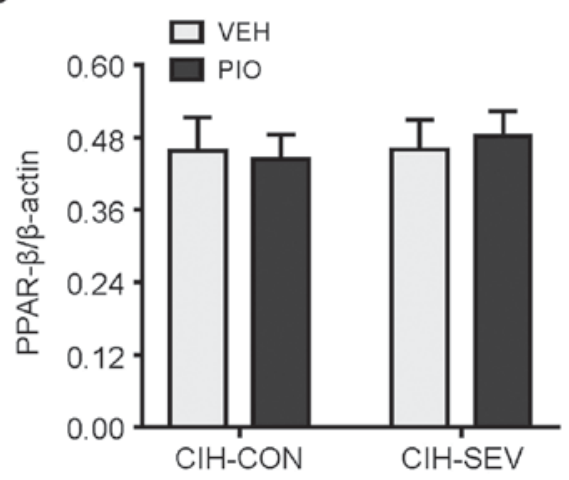

Figure 5. Effects of pioglitazone (PIO) treatment on expression of PPAR isoforms PPAR- $\alpha$ (A and B) and PPAR- $\beta$ (A and C) in the hippocampus in the $\mathrm{CIH}$ rats. There were no differences in expression of PPAR- $\alpha$ or PPAR- $\beta$ among the four groups. Data are expressed as the mean \pm SEM ( $=9$ per group). PPAR, peroxisome proliferator-activated receptor; $\mathrm{CIH}$, chronic intermittent hypoxia; CON, control; VEH, vehicle.

impair cognitive function under physiological conditions, but it induced cognitive decline under CIH conditions (28-30). In the present study, it was found that CIH-SEV rats treated with VEH exhibited decline in both spatial learning and memory in the Morris Water Maze task when compared with CIH-CON rats treated with $\mathrm{VEH}$. These observations are consistent with previous reports (28), suggesting that a moderate duration of SEV causes cognitive decline in animals under $\mathrm{CIH}$ conditions.

Our previous study (28) demonstrated that SEV exaggerated microglia activity and neuroinflammation in the hippocampus, which were associated with reductions in hippocampal PPAR- $\gamma$ expression and activity. In the present study, the number of activated microglia and proinflammatory cytokine expression were increased but PPAR- $\gamma$ expression and activity were decreased in the hippocampus in CIH-SEV rats treated with $\mathrm{VEH}$, compared with $\mathrm{CIH}-\mathrm{CON}$ rats treated with VEH. These results confirm previous findings, suggesting that downregulation of PPAR- $\gamma$ in the hippocampus by SEV might contribute to exaggerated neuroinflammation and consequently cognitive decline by increasing the sensitivity and susceptibility to $\mathrm{CIH}$ insult.

Experimental studies in animals have reported that systemic treatment with PIO attenuates brain microglial activation and neuroinflammation, improves neuronal survival, and prevents cognitive impairment in many inflammatory central nervous system disorders, such as chronic stress (32), alcohol-induced neuronal damage (31), traumatic brain injury (35) and forebrain ischemia/reperfusion injury (36). In the present study, systemic treatment with PIO, starting one day prior to SEV exposure, markedly increased PPAR- $\gamma$ expression and activity in the hippocampus of CIH-SEV rats, which were associated with significant reductions in microglia activity and proinflammatory cytokine expression. Moreover, systemic treatment with PIO also improved cognitive decline in CIH-SEV rats as indicated by decreased latency to find the hidden platform and increased the percent time spent in the target quadrant, suggesting that SEV exposure causes a decline in spatial learning and memory abilities under $\mathrm{CIH}$ conditions, which can be prevented by treatment with PPAR- $\gamma$ agonist PIO. Of note, swimming speed was similar among the 4 experimental groups, indicating that no alteration in sensory-motor activity that would influence the Morris Water Maze performance was produced by either SEV exposure or PIO treatment. Importantly, systemic treatment with PIO also markedly increased PPAR $-\gamma$ expression and activity in the hippocampus of CIH-CON rats but did not alter microglia activity and proinflammatory cytokine expression as well as cognitive function in these animals, suggesting that microglia activity and proinflammatory cytokine expression in the hippocampus of CIH-CON rats without SEV exposure are mediated by other mechanisms rather than PPAR- $\gamma$. However, SEV exposure reduced PPAR- $\gamma$ expression and activity, which increased sensitivity to inflammatory stimuli, resulting in exaggerated inflammatory responses. Collectively, these results indicate that systemic treatment with PIO inhibits SEV exposure-induced exaggeration of microglia-mediated neuroinflammation and cognitive decline in $\mathrm{CIH}$ rats via upregulation hippocampal PPAR $-\gamma$. PPAR- $\gamma$ can physically bind to NF- $\kappa \mathrm{B}$ p65 to inhibit NF- $\kappa \mathrm{B}$ activity $(37,38)$, and SEV exposure has been reported to induce microglia activation and neuroinflammation through the $\mathrm{NF}-\kappa \mathrm{B}$ pathway $(39,40)$. Thus, the salutary effects of PIO treatment in the brain of CIH-SEV rats may be explained by the ability of PPAR $-\gamma$ to inhibit SEV-induced NF- $\kappa \mathrm{B}$ activity. 
It is worth noting that PPAR- $\alpha$ and PPAR- $\beta$, two other PPAR isotypes, are also expressed in the brain and have been shown to exert anti-inflammatory and neuroprotective actions (41). However, expression of PPAR- $\alpha$ and PPAR- $\beta$ in the hippocampus was similar among the 4 groups, excluding the possibility that PIO treatment inhibited microglia-mediated neuroinflammation in CIH-SEV rats by modulation of PPAR- $\alpha$ and PPAR- $\beta$.

One limitation of the study should be acknowledged. In the present study, we measured PPAR- $\gamma$ expression and activity in the hippocampus but did not examine any PPAR- $\gamma$ downstream targets. Further studies are necessary to determine whether PPAR $-\gamma$ downstream targets are involved in the beneficial effects of PIO treatment on SEV-induced cognitive dysfunction under $\mathrm{CIH}$ conditions and provide detailed mechanisms.

In summary, the present study demonstrated that treatment with PPAR- $\gamma$ agonist PIO prevents anesthetic SEV-induced cognitive decline in $\mathrm{CIH}$ rats and that these beneficial effects are mediated by upregulation of PPAR- $\gamma$, which inhibits SEV-induced neuroinflammation in the hippocampus. These findings may provide new insight into the potential use of PIO for preventing anesthetic SEV-induced cognitive decline in surgical patients with obstructive sleep apnea.

\section{Acknowledgements}

Not applicable.

\section{Funding}

This study was supported by the Major Project of Science and Technology of Shandong Province (no. 2016GSF201070 to DL).

\section{Availability of data and materials}

The datasets used and/or analyzed during the present study are available from the corresponding author on reasonable request.

\section{Authors' contribution}

$\mathrm{XZ}$ and JF designed the experiments and wrote the manuscript. XZ, NL, LLu, QL, LLi, PD, BY and DL performed the experiments, collected and analyzed the data. All authors read and approved the manuscript and agree to be accountable for all aspects of the research in ensuring that the accuracy or integrity of any part of the work are appropriately investigated and resolved.

\section{Ethics approval and consent to participate}

All experimental protocols were approved by the Animal Care and Use Committees of Shandong University and conducted in accordance with the guidelines of the Animal Care and Use Committee at Shandong University (Jinan, Shandong, China).

\section{Patient consent for publication}

Not applicable.

\section{Competing interests}

The authors declare that they have no conflict of interest.

\section{References}

1. Skvarc DR, Berk M, Byrne LK, Dean OM, Dodd S, Lewis M, Marriott A, Moore EM, Morris G, Page RS and Gray L: Post-operative cognitive dysfunction: An exploration of the inflammatory hypothesis and novel therapies. Neurosci Biobehav Rev 84: 116-133, 2018.

2. Jevtovic-Todorovic V, Absalom AR, Blomgren K, Brambrink A, Crosby G, Culley DJ, Fiskum G, Giffard RG, Herold KF, Loepke AW, et al: Anaesthetic neurotoxicity and neuroplasticity: An expert group report and statement based on the BJA Salzburg Seminar. Br J Anaesth 111: 143-151, 2013.

3. Callaway JK, Jones NC, Royse AG and Royse CF: Memory impairment in rats after desflurane anesthesia is age and dose dependent. J Alzheimers Dis 44: 995-1005, 2015.

4. Shen X, Dong Y, Xu Z, Wang H, Miao C, Soriano SG, Sun D, Baxter MG, Zhang Y and Xie Z: Selective anesthesia-induced neuroinflammation in developing mouse brain and cognitive impairment. Anesthesiology 118: 502-515, 2013.

5. Feng X, Degos V, Koch LG, Britton SL, Zhu Y, Vacas S, Terrando N, Nelson J, Su X and Maze M: Surgery results in exaggerated and persistent cognitive decline in a rat model of the metabolic syndrome. Anesthesiology 118: 1098-1105, 2013.

6. Yang C, Zhu B, Ding J and Wang ZG: Isoflurane anesthesia aggravates cognitive impairment in streptozotocin-induced diabetic rats. Int J Clin Exp Med 7: 903-910, 2014.

7. Yue T, Shanbin G, Ling M, Yuan W, Ying X and Ping Z: Sevoflurane aggregates cognitive dysfunction and hippocampal oxidative stress induced by $\beta$-amyloid in rats. Life Sci 143: 194-201, 2015

8. Wadhwa M, Prabhakar A, Ray K, Roy K, Kumari P, Jha PK, Kishore K, Kumar S and Panjwani U: Inhibiting the microglia activation improves the spatial memory and adult neurogenesis in rat hippocampus during $48 \mathrm{~h}$ of sleep deprivation. J Neuroinflammation 14: 222, 2017.

9. Li Z, Ni C, Xia C, Jaw J, Wang Y, Cao Y, Xu M and Guo X: Calcineurin/nuclear factor- $\kappa \mathrm{B}$ signaling mediates isoflurane-induced hippocampal neuroinflammation and subsequent cognitive impairment in aged rats. Mol Med Rep 15: 201-209, 2017.

10. Cai JC, Liu W, Lu F, Kong WB, Zhou XX, Miao P, Lei CX and Wang Y: Resveratrol attenuates neurological deficit and neuroinflammation following intracerebral hemorrhage. Exp Ther Med 15: 4131-4138, 2018

11. Block ML: Neuroinflammation: Modulating mighty microglia. Nat Chem Biol 10: 988-989, 2014.

12. Liu H, Huang F, Wu H, Zhang B, Shi H, Wu X and Hu Z: Isoastragaloside I inhibits NF- $\kappa \mathrm{B}$ activation and inflammatory responses in BV-2 microglial cells stimulated with lipopolysaccharide. Int J Mol Med 40: 1270-1276, 2017.

13. Jin M, Park SY, Shen Q, Lai Y, Ou X, Mao Z, Lin D, Yu Y and Zhang W: Anti-neuroinflammatory effect of curcumin on Pam3CSK4-stimulated microglial cells. Int J Mol Med 41: 521-530, 2018.

14. Zhang Z, Yuan H, Zhao H, Qi B, Li F and An L: PPAR $\gamma$ activation ameliorates postoperative cognitive decline probably through suppressing hippocampal neuroinflammation in aged mice. Int Immunopharmacol 43: 53-61, 2017.

15. Gagnon K, Baril AA, Gagnon JF, Fortin M, Décary A, Lafond C, Desautels A, Montplaisir J and Gosselin N: Cognitive impairment in obstructive sleep apnea. Pathol Biol (Paris) 62: 233-240, 2014.

16. Jin F, Liu J, Zhang X, Cai W, Zhang Y, Zhang W, Yang J, Lu G and Zhang X: Effect of continuous positive airway pressure therapy on inflammatory cytokines and atherosclerosis in patients with obstructive sleep apnea syndrome. Mol Med Rep 16: 6334-6339, 2017.

17. Sforza E and Roche F: Chronic intermittent hypoxia and obstructive sleep apnea: An experimental and clinical approach. Hypoxia (Auckl) 4: 99-108, 2016.

18. Yaffe K, Laffan AM, Harrison SL, Redline S, Spira AP, Ensrud KE, Ancoli-Israel S and Stone KL: Sleep-disordered breathing, hypoxia, and risk of mild cognitive impairment and dementia in older women. JAMA 306: 613-619, 2011. 
19. Moore-Carrasco R, Poblete Bustamante M, González Guerra O, Leiva Madariaga E, Mujica Escudero V, Aranguez Arellano C and Palomo I: Peroxisome proliferator-activated receptors: Targets for the treatment of metabolic illnesses (Review). Mol Med Rep 1: 317-324, 2008.

20. Martin H: Role of PPAR-gamma in inflammation. Prospects for therapeutic intervention by food components. Mutat Res 690: $57-63,2010$

21. Zhang T, Shao B and Liu GA: Rosuvastatin promotes the differentiation of peripheral blood monocytes into M2 macrophages in patients with atherosclerosis by activating PPAR- $\gamma$. Eur Rev Med Pharmacol Sci 21: 4464-4471, 2017.

22. Wang Q, Su YY, Li YQ, Zhang YF, Yang S, Wang JL and Li HY: Atorvastatin alleviates renal ischemia-reperfusion injury in rats by promoting M1-M2 transition. Mol Med Rep 15: 798-804, 2017.

23. Culman J, Zhao Y, Gohlke P and Herdegen T: PPAR-gamma: Therapeutic target for ischemic stroke. Trends Pharmacol Sci 28: 244-249, 2007.

24. Kapadia R, Yi JH and Vemuganti R: Mechanisms of anti-inflammatory and neuroprotective actions of PPAR-gamma agonists. Front Biosci 13: 1813-1826, 2008.

25. Nicolakakis $\mathrm{N}$ and Hamel E: The nuclear receptor PPARgamma as a therapeutic target for cerebrovascular and brain dysfunction in Alzheimer's disease. Front Aging Neurosci 2: 21, 2010.

26. Domi E, Uhrig S, Soverchia L, Spanagel R, Hansson AC, Barbier E, Heilig M, Ciccocioppo R and Ubaldi M: Genetic deletion of neuronal PPAR $\gamma$ enhances the emotional response to acute stress and exacerbates anxiety: An effect reversed by rescue of amygdala PPAR $\gamma$ Function. J Neurosci 36: 12611-12623, 2016.

27. Bernardo A and Minghetti L: Regulation of glial cell functions by PPAR-gamma natural and synthetic agonists. PPAR Res 2008: 864140, 2008.

28. Dong P, Zhao J, Li N, Lu L, Li L, Zhang X, Yang B, Zhang L and Li D: Sevoflurane exaggerates cognitive decline in a rat model of chronic intermittent hypoxia by aggravating microglia-mediated neuroinflammation via downregulation of PPAR- $\gamma$ in the hippocampus. Behav Brain Res 347: 325-331, 2018.

29. Callaway JK, Jones NC, Royse AG and Royse CF: Sevoflurane anesthesia does not impair acquisition learning or memory in the Morris water maze in young adult and aged rats. Anesthesiology 117: 1091-1101, 2012

30. Li D, Liu L, Li L, Li X, Huang B, Zhou C, Zhang Z, Wang C, Dong P, Zhang X, et al: Sevoflurane induces exaggerated and persistent cognitive decline in a type II diabetic rat model by aggregating hippocampal inflammation. Front Pharmacol 8: 886, 2017.

31. Cippitelli A, Domi E, Ubaldi M, Douglas JC, Li HW, Demopulos G, Gaitanaris G, Roberto M, Drew PD, Kane CJ and Ciccocioppo R: Protection against alcohol-induced neuronal and cognitive damage by the PPAR $\gamma$ receptor agonist pioglitazone. Brain Behav Immun 64: 320-329, 2017.
32. Zhao Q, Wu X, Yan S, Xie X, Fan Y, Zhang J, Peng C and You Z: The antidepressant-like effects of pioglitazone in a chronic mild stress mouse model are associated with PPAR $\gamma$-mediated alteration of microglial activation phenotypes. J Neuroinflammation 13 : 259, 2016.

33. Grommes C, Karlo JC, Caprariello A, Blankenship D, Dechant A and Landreth GE: The PPAR $\gamma$ agonist pioglitazone crosses the blood-brain barrier and reduces tumor growth in a human xenograft model. Cancer Chemother Pharmacol 71: 929-936, 2013.

34. Garvey JF, Pengo MF, Drakatos P and Kent BD: Epidemiological aspects of obstructive sleep apnea. J Thorac Dis 7: 920-929, 2015.

35. Liu M, Bachstetter AD, Cass WA, Lifshitz J and Bing G: Pioglitazone attenuates neuroinflammation and promotes dopaminergic neuronal survival in the nigrostriatal system of rats after diffuse brain injury. J Neurotrauma 34: 414-422, 2017.

36. Collino M, Aragno M, Mastrocola R, Gallicchio M, Rosa AC, Dianzani C, Danni O, Thiemermann C and Fantozzi R: Modulation of the oxidative stress and inflammatory response by PPAR-gamma agonists in the hippocampus of rats exposed to cerebral ischemia/reperfusion. Eur J Pharmacol 530: 70-80, 2006.

37. Chen F, Wang M, O'Connor JP, He M, Tripathi T and Harrison LE: Phosphorylation of PPARgamma via active ERK1/2 leads to its physical association with $\mathrm{p} 65$ and inhibition of NF-kappabeta. J Cell Biochem 90: 732-744, 2003.

38. Ruan H, Pownall HJ and Lodish HF: Troglitazone antagonizes tumor necrosis factor-alpha-induced reprogramming of adipocyte gene expression by inhibiting the transcriptional regulatory functions of NF-kappaB. J Biol Chem 278: 28181-28192, 2003.

39. Wang W, Chen X, Zhang J, Zhao Y, Li S, Tan L, Gao J, Fang X and Luo A: Glycyrrhizin attenuates isoflurane-induced cognitive deficits in neonatal rats via its anti-inflammatory activity. Neuroscience 316: 328-336, 2016.

40. Zhang L, Zhang J, Yang L, Dong Y, Zhang Y and Xie Z: Isoflurane and sevoflurane increase interleukin-6 levels through the nuclear factor-kappa B pathway in neuroglioma cells. Br J Anaesth 110 (Suppl 1): i82-i91, 2013.

41. Warden A, Truitt J, Merriman M, Ponomareva O, Jameson K, Ferguson LB, Mayfield RD and Harris RA: Localization of PPAR isotypes in the adult mouse and human brain. Sci Rep 6: 27618, 2016. 